\title{
Cluster-based optimization of cellular materials and structures for crashworthiness
}

\author{
This paper is intended for the special issue on "Design of Engineered Materials and Structures"
}

\author{
Kai Liu \\ School of Mechanical Engineering \\ Purdue University \\ West Lafayette, Indiana, USA \\ liu915@purdue.edu \\ Duane Detwiler \\ Honda R\&D Americas, Inc. \\ Raymond, Ohio, USA \\ ddetwiler@oh.hra.com \\ Andres Tovar* \\ Associate Professor \\ Department of Mechanical and Energy Engineering \\ Purdue School of Engineering and Technology \\ Indiana University-Purdue University Indianapolis \\ Indianapolis, Indiana, USA \\ tovara@iupui.edu
}

The objective of this work is to establish a cluster-based optimization method for the optimal design of cellular materials and structures for crashworthiness, which involves the use of nonlinear, dynamic finite element models. The proposed method uses a cluster-based structural optimization approach consisting of four steps: conceptual design generation, clustering, metamodel-based global optimization, and cellular material design. The conceptual design is generated using structural optimization methods. K-means clustering is applied to the conceptual design to reduce the dimensional of the design space as well as define the internal architectures of the multimaterial structure. With reduced dimension space, global optimization aims to improve the crashworthiness of the structure can be performed efficiently. The cellular material design incorporates two homogenization methods, namely, energy-based homogenization for linear and nonlinear elastic material models and mean-field homogenization for (fully) nonlinear material models. The proposed methodology is demonstrated using three designs for crashworthiness that include linear, geometrically nonlinear and nonlinear models.

*Address all correspondence to this author.

\section{Introduction}

A cellular material is an assembly of unit cells, which are themselves (mesoscale) structures formed by solid and void phases. According to the unit cell architecture, cellular materials can be classified into (two-dimensional) honeycombs and (three-dimensional) foams [1]. These materials are observed in nature in the form of wood, cork, trabecular bone, plant parenchyma, and marine sponges, among others [2]. In engineering, man-made honeycombs and foams are used in a variety of applications raging from coffee cups and lattice injection molds [3] to helmet liners [4] and crash padding in automotive vehicles [5]. Design of cellular materials and (macroscale) cellular structures is gaining relevance due to advances in additive manufacturing and structural optimization methods. Models for functionally graded polymeric foams have proposed in order to improve the energy absorption characteristics offered by uniform foams [6]. The application of metal foams in crashworthiness has been increasingly considered as fillers of straight [7-10] and tapered [11-13] thin-walled tubular structures. Most of these studies rely on experimental testing and numerical simulations. Using analytical expressions of honeycomb unit cells, negative stiffness honeycomb structures have been designed for lightweight stiffness and energy absorption [14-16]. Multi- 
scale design of negative stiffness honeycombs have been also addressed with Bayesian network classifiers [17].

Topology optimization of cellular materials can be dated to the seminal work from Bendsøe and Kikuchi [18] used two-dimensional square voids (honeycomb-like material) in conjunction with the homogenization method to minimize structural compliance. A hierarchical topology optimization method has been proposed in which the topology of the cellular material unit cell is optimized using the inverse homogenization method [19]. Their work has been extended to include 3D cellular materials [20] and hyperelastic material models [21].

The traditional homogenization and inverse homogenization methods used in topology optimization can be efficiently applied to the design of cellular materials and structures with linear material models; unfortunately, such methods are of limited application in design for crashworthiness due to the nonlinear natural of the models, which include large strains, plasticity, and contact. To address this limitation, multiscale analysis methods like $\mathrm{FE}^{2}$ [22-24] can be applied. In this method, each point of the (macroscopic) structure is associated with a cellular unit cell and, for each macroscopic equilibrium iteration, a nonlinear load increment needs to be computed for each of the cellular unit cells. While promising, this approach is computationally expensive and not easily scalable to $3 \mathrm{D}$ crash structures undergoing dynamic loads. A recent review of design optimization methods for structural crashworthiness has been presented by [25].

The objective of this work is to propose a cluster-based optimization method for the optimal design of cellular materials and structures that involving nonlinear dynamic crashworthiness analysis. The proposed method builds upon a cluster-based structural optimization approach previously presented by the authors [26]. The basic approach consists of three steps: conceptual design generation, clustering, and metamodel-based global optimization. The method proposed in this work extends the basic approach by incorporating a fourth step consisting on the design of the cellular material. The cellular material design makes use of two homogenization methods, namely, energy-based homogenization [27,28] for linear and nonlinear elastic material models, and meanfield homogenization [29, 30] for nonlinear material models. The result of the metamodel-based global optimization is the Pareto optimal cellular structures. For elastic material models, inverse homogenization problems are solved to synthesize the topology of the cellular unit cell of each material cluster. For nonlinear material models, the cellular material design step is incorporated into the metamodel-based global optimization by applying the mean-field homogenization method on predefined, parameterized cellular unit cells.

This paper is organized as follows. The basic clusteredbased structural optimization method is summarized in Sec. 2. The proposed method for design of cellular materials is presented in Sec. 3. Numerical examples that include linear, geometrically nonlinear, and (fully) nonlinear models are illustrated in Sec. 4. Finally, a summary and discussion are presented in Sec. 5 .

\section{Cluster-based Structural Optimization}

The basic cluster-based structural optimization approach involves three design steps: conceptual design generation, clustering, and metamodel-based global optimization. The method is presented in detail in [26] and summarized in this section for clarity.

\subsection{Conceptual design (Step 1)}

The first step in the proposed design strategy is to generate a conceptual multimaterial design by solving a (related, but simpler) structural optimization problem

$$
\begin{aligned}
\text { find } & \mathbf{x} \in \mathbb{R}^{n} \\
\text { minimize } & f(\mathbf{x}, \mathbf{U}(\mathbf{x})) \\
\text { subject to } & \mathbf{h}(\mathbf{x}, \mathbf{U}(\mathbf{x}))=\mathbf{0} \\
& \mathbf{g}(\mathbf{x}, \mathbf{U}(\mathbf{x})) \leqslant \mathbf{0} \\
& 0 \leqslant x_{e} \leqslant 1, \quad e=1, \ldots, n,
\end{aligned}
$$

satisfying the finite element equilibrium equations. In this problem, the material properties are characterized by the design variables $x_{e} \in \mathbb{R}$, for $e=1, \ldots, n$, where $n$ is the number of finite elements in the design domain, $f$ is the objective function, $\mathbf{h}(\mathbf{x}, \mathbf{U}(\mathbf{x}))$ are the equality constraints, $\mathbf{g}(\mathbf{x}, \mathbf{U}(\mathbf{x}))$ are the inequality constraints, and $\mathbf{U}(\mathbf{x})$ are the global nodal displacements.

To generate the conceptual design, this work makes use of the Voigt upper bound material mixture model:

$$
\mathrm{E}_{e}\left(x_{e}\right)=\mathrm{E}_{\min }+\left(\mathrm{E}_{0}-\mathrm{E}_{\min }\right) x_{e}
$$

where $\mathrm{E}_{e}$ is the element material Young's modulus and $\mathrm{E}_{0}$ is the base material Young's modulus. The conceptual material distribution $\mathbf{x}^{*} \in \mathbb{R}^{n}$ from (1) is used as the conceptual design. In practice, the desired number of desired materials $K$ in the final design is orders of magnitude lower than $n$; therefore, the dimension of the problem is reduced as described in Sec. 2.2.

\subsection{Clustering (Step 2)}

The dimension reduction problem is addressed by cluster analysis, which leads to groups of observations in such a way that the observations in the same group are more similar to each other than to those in other groups. This work uses K-means [31], but other cluster analysis methods can be implemented.

The input observations to the cluster analysis algorithm is the conceptual material distribution $\mathbf{x}^{*} \in \mathbb{R}^{n}$ and the desired number of clusters $K$, where $1 \leqslant K \leqslant n$; usually, $K \ll n$ (Algorithm 1 [32]). A discussion on the optimal value of $K$ can be found in [33].

The iterative refinement clustering algorithm starts by distributing $K$ cluster centers $\mu_{k}(k=1, \ldots, K)$ within the set of material parameters $x_{e}^{*}(e=1, \ldots, n)$. According to the distance between $x_{e}^{*}$ and $\mu_{k}$, the set is partitioned into $K$ Voronoi 
clusters $S_{k}$. From this point, the algorithm finds the cluster centers that minimize the sum of square distance as follows:

$$
\begin{aligned}
\text { find } & \boldsymbol{\mu} \in \mathbb{R}^{K} \\
\operatorname{minimize} & J(\boldsymbol{\mu})=\sum_{k=1}^{K} \sum_{x_{e}^{*} \in S_{k}}\left(x_{e}^{*}-\mu_{k}\right)^{2},
\end{aligned}
$$

where the K-means objective function $J(\boldsymbol{\mu})$ is referred to as the within-cluster sum of squares. The K-means clustering algorithm converges when the cluster centers no longer change. The result is a $K$-dimensional clustered design suitable for building metamodels and performing global optimization as described in Sec. 2.3.

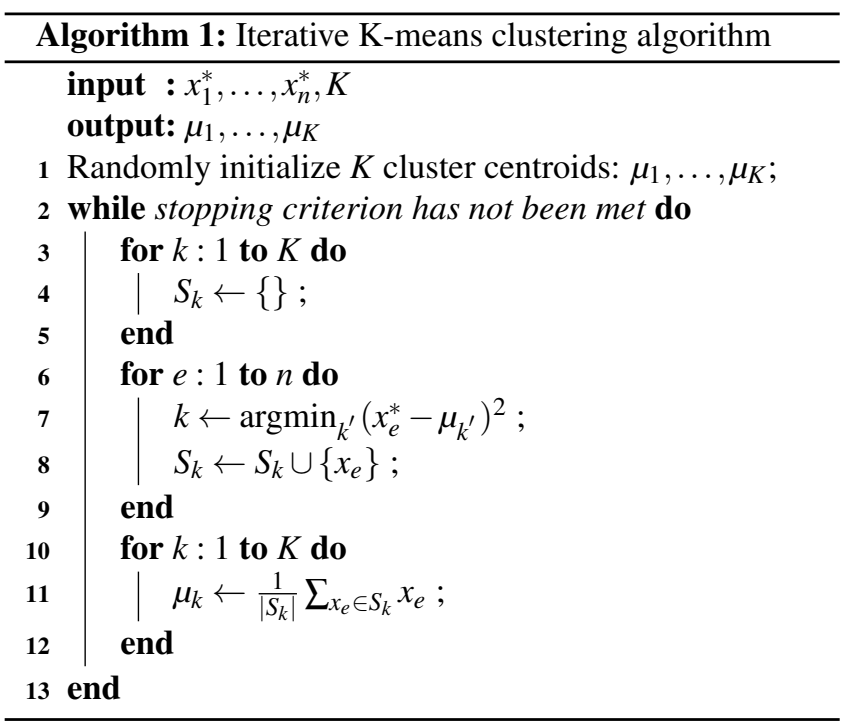

\subsection{Metamodel-based global optimization (Step 3)}

A relevant aspect of using metamodels in global optimization is the ability to balance global exploration and local exploitation. In other words, it is desirable to generate an accurate metamodel that explores a large portion of the design space with a few sampling points [34]. Several metamodels have been evaluated for design problems in crashworthiness, including: polynomial response surface, radial basis functions, and Kriging [33]. Based on cross-validation errors, Kriging is the preferred metamodel and it is used in this work. A Kriging metamodel $\hat{f}$ of a scalar-valued function $f$ at the prediction point $\mathbf{S}^{(p)}$ is given by

$$
\hat{f}(\mathbf{S})=\mathrm{E}[\hat{f}(\mathbf{S})]+\sum_{p=1}^{P} \omega_{p}\left\{f\left(\mathbf{S}^{(p)}\right)-\mathrm{E}\left[f\left(\mathbf{S}^{(p)}\right)\right]\right\}
$$

where $\mathrm{E}[\cdot]$ is the expected value (mean), $\mathbf{S}^{(p)}$ are the $p$ th sampled designs, and $\omega_{p}$ are the Kriging weights, which are derived from a covariance function. This metamodel can be found implemented in MATLAB [35].
The input to the metamodels $\hat{\mathbf{f}}$ is $\boldsymbol{\mu}$. The output is the predicted values of the functions $\mathbf{f}$. One metamodel is built for each function in the optimization problem using Latin hypercube sampling (LHS) points. Once the metamodels are built, the global optimization problem can be solved. The global optimization problem is to find the material parameters that minimize the objective function vector $\hat{\mathbf{f}}(\mathbf{x}(\boldsymbol{\mu})): \mathbb{R}^{K} \rightarrow \mathbb{R}^{n_{f}}$, where $n_{f}$ is the number of objective functions. This is,

$$
\begin{aligned}
\text { find } & \boldsymbol{\mu} \in \mathbb{R}^{K} \\
\text { minimize } & \hat{\mathbf{f}}(\mathbf{x}(\boldsymbol{\mu})) \\
\text { subject to } & \mu_{k}^{L} \leqslant \mu_{k} \leqslant \mu_{k}^{U} \\
& k=1, \ldots, K .
\end{aligned}
$$

where $\boldsymbol{\mu}^{L}$ and $\boldsymbol{\mu}^{U}$ is a vector of lower and upper bounds on the clustered design variables $\boldsymbol{\mu}$, respectively. The optimal design can be found using the Efficient Global Optimization (EGO) algorithm [34,36]. During the search for the global optimum, the EGO algorithm balances between global exploration and local exploitation by using the expected improvement function. The expected improvement function calculates the amount of improvement one can expect at a given point $\mathbf{S}^{*(p)}$ : consider an optimization problem that minimizes two objectives $f_{1}(\mathbf{x})$ and $f_{2}(\mathbf{x})$, with the set of $m$ Pareto points

$$
\mathbf{f}_{1,2}^{*}=\left\{\left(f_{1}^{*(1)}, f_{2}^{*(1)}\right), \ldots,\left(f_{1}^{*(m)}, f_{2}^{*(m)}\right)\right\}
$$

where $f_{j}^{*(i)}=f_{j}\left(\mathbf{S}^{*(i)}\right)$ and $\mathbf{S}^{*(i)}$ is a Pareto design. The expected improvement for this multi-objective problem is defined as [34]:

$$
\mathrm{E}\left[I\left(\mathbf{S}^{*(p)}\right)\right]=\mathrm{P}\left[I\left(\mathbf{S}^{*(p)}\right)\right] \min \left\{d_{1}, \ldots, d_{m}\right\},
$$

where $\mathrm{P}\left[I\left(\mathbf{S}^{*(p)}\right)\right]$ is the probability of improving both functions $f_{1}$ and $f_{2}$ at the Pareto design $\mathbf{S}^{*(p)}$. The probability of improvement is defined as:

$$
\begin{array}{r}
\mathrm{P}\left[I\left(\mathbf{S}^{*(p)}\right)\right]=\Phi\left(u_{1}^{1}\right)+\sum_{i=1}^{m-1}\left[\Phi\left(u_{1}^{i+1}\right)-\Phi\left(u_{1}^{i}\right)\right] \Phi\left(u_{2}^{i+1}\right)+ \\
{\left[1-\Phi\left(u_{1}^{m}\right)\right] \Phi\left(u_{2}^{m}\right),}
\end{array}
$$

where $u_{j}^{i}=u_{j}^{i}\left(\mathbf{S}^{*(p)}\right)=\left(f_{j}^{*(i)}-\hat{f}_{j}\left(\mathbf{S}^{*(p)}\right)\right) / \sigma_{j}\left(\mathbf{S}^{*(p)}\right)$.

In (7), $d_{i}$ for $i=1, \ldots, m$ is the distance between the vectors $\left(\bar{F}_{1}, \bar{F}_{2}\right)$ and $\left(f_{1}^{*(i)}, f_{2}^{*(i)}\right)$, where $\left(\bar{F}_{1}, \bar{F}_{2}\right)$ is the centroid of the probability integral used to calculate $\mathrm{E}\left[I\left(\mathbf{S}^{*(p)}\right)\right]$ :

$$
\begin{array}{r}
\bar{F}_{1}\left(\mathbf{S}^{*(p)}\right)=\frac{1}{\mathrm{P}\left[I\left(\mathbf{S}^{*(p)}\right)\right]}\left[z_{1}^{1}+\sum_{i=1}^{m-1}\left(z_{1}^{i+1}-z_{1}^{i}\right) \Phi\left(u_{2}^{i+1}\right)+\right. \\
\left.z_{1}^{m} \Phi\left(u_{2}^{m}\right)\right]
\end{array}
$$


where $z_{j}^{i}=z_{j}^{i}\left(\mathbf{S}^{*(p)}\right)=\hat{f}_{j}\left(\mathbf{S}^{*(p)}\right) \Phi\left(u_{j}^{i}\right)-\sigma_{j}\left(\mathbf{S}^{*(p)}\right) \phi\left(u_{j}^{i}\right)$. $\bar{F}_{2}\left(\mathbf{S}^{*(p)}\right)$ is defined similarly. Details on the derivation of the multi-objective expected improvement formula can be found in [34].

The expected improvement function is maximized using an evolutionary algorithm. If the maximum expected improvement is less than $0.1 \%$ of the present best function value in two consecutive iterations, then convergence is achieved and the metamodel needs no further improvement; otherwise, the point where the expected improvement is maximized is added to the sampled set and the metamodel is updated. If no convergence is achieved in 100 iterations, the algorithm is terminated.

\section{Design of cellular materials}

The basic cluster-based structural optimization approach (Sec. 2) is extended in this work to the design of cellular materials and structures. The focus is on quasi-periodic lattice structures in which each cellular material is a periodic, lattice array. The proposed approach is suitable for both linear and nonlinear cellular material models. For the design of linear cellular structures, this work implements inverse homogenization so an optimal cellular unit cell topology can be synthesized. For nonlinear cellular structures, mean-field homogenization is implemented so that nonlinear cellular material properties can be approximated.

\subsection{Mean field homogenization of nonlinear cellular structure properties}

Mean-field homogenization estimates the volume averages of the stress and strain fields avoiding the use of finite element analysis [29]. For a two-phase material, a field volume average over a unit cell is estimated as follows:

$$
\left\langle g\left(\mathbf{x}_{e}\right)\right\rangle=v_{0}\left\langle g\left(\mathbf{x}_{e}\right)\right\rangle_{\omega_{0}}+v_{1}\left\langle g\left(\mathbf{x}_{e}\right)\right\rangle_{\omega_{1}},
$$

where $\langle\cdot\rangle$ is the averaging operator, $g\left(\mathbf{x}_{e}\right)$ is any field (e.g., stress or strain) defined over the unit cell, $\omega_{0}$ and $\omega_{1}$ are the material phases, and $v_{1}$ and $v_{0}$ are corresponding the volume fractions, such that $v_{1}+v_{0}=1$.

Mean-field homogenization models include Voigt (uniform strain), Reuss (uniform stress), Self-Consistent model, Mori-Tanaka model, Double inclusion model, Lielens interpolation model, and Bound interpolation model [29,30]. For any of these models, two dependent strain concentration tensors, $\mathbf{A}^{\varepsilon}$ and $\mathbf{B}^{\varepsilon}$, are defined according to

$$
\langle\varepsilon\rangle_{\omega_{0}}=\mathbf{B}^{\varepsilon}:\langle\varepsilon\rangle_{\omega_{1}}, \quad\langle\varepsilon\rangle_{\omega_{0}}=\mathbf{A}^{\varepsilon}:\langle\varepsilon\rangle_{\omega},
$$

where $\omega=\omega_{0} \cup \omega_{1}$. This work uses the Mori-Tanka model [37]. This model is based on a two-phase composite (matrix and inclusion) and the solution to the Eshelby's problem [38]. The corresponding strain concentration tensor is given by:

$$
\mathbf{B}^{\varepsilon}=\left\{\mathbf{I}+\xi\left(I, \mathbf{C}_{1}\right): \mathbf{C}_{1}^{-1}:\left[\mathbf{C}_{0}-\mathbf{C}_{1}\right]\right\}^{-1}
$$

where $\xi\left(I, \mathbf{C}_{1}\right)$ is Eshelby's tensor, $\mathbf{C}_{1}$ and $\mathbf{C}_{0}$ are the uniform stiffness tensors of the matrix and the inclusion, respectively, and $\mathbf{I}$ is the fourth-rank symmetric identity tensor.

The generalization of the Eshelby's solution and the Mori-Tanaka homogenization model from linear elasticity to nonlinear elasto-plasticity involves the incremental linearization of the stress rate and the strain rate fields at each material point in each phase through a tangent operator $\mathbf{C}_{r}$ as follows:

$$
\dot{\boldsymbol{\sigma}}(\mathbf{X}, t)=\mathbf{C}_{r}(\varepsilon(\mathbf{X}, t), t): \dot{\varepsilon}(\mathbf{X}, t),
$$

where $t$ is the time and $\mathbf{X}$ is the spatial coordinate of the material point. Notably, $\mathbf{C}_{r}$ is not uniform, i.e., the stress and strain fields are not uniform within the phase. Therefore, the Eshelby's solution and the Mori-Tanaka homogenization model cannot be applied [37,38]. In order to homogenize (13), comparison materials that are uniform within the phase can be defined as

$$
\dot{\boldsymbol{\sigma}}(\mathbf{X}, t)=\hat{\mathbf{C}}_{r}(t): \dot{\varepsilon}(\mathbf{X}, t)
$$

where $\hat{\mathbf{C}}_{r}(t)$ is the approximate tangent operator for a uniform material. The definition of $\hat{\mathbf{C}}_{r}(t)$ and the practical implementations of the nonlinear mean-field homogenization method are discussed in details in [30]. This work uses DigIMAT-MF to perform the mean-field homogenization.

\subsection{Energy-based homogenization of linear cellular structure properties}

For linear elastic materials, the homogenized stiffness tensor $C_{i j k l}^{H}$ of the cellular materials can be calculated using the homogenization theory $[39,40]$ by integrating over the base cell volume $Y$,

$$
C_{i j k l}^{H}=\frac{1}{|Y|} \int_{Y} C_{i j p q}\left(\varepsilon_{p q}^{0(k l)}-\varepsilon_{p q}^{*(k l)}\right) d Y,
$$

where $\varepsilon_{p q}^{*(k l)}$ is the periodic solution of

$$
\begin{aligned}
\int_{Y} C_{i j p q} \varepsilon_{p q}^{*(k l)} \frac{\partial v_{i}}{\partial y_{j}} d Y & =\int_{Y} C_{i j p q} \varepsilon_{p q}^{0(k l)} \frac{\partial v_{i}}{\partial y_{j}} d Y, \forall v \in V \\
V & =\{v: v \text { is Y-periodic }\}
\end{aligned}
$$

where $\varepsilon_{p q}^{0(k l)}$ is the unit test strain fields. (15) can be rewritten in terms of mutual energies [40]:

$$
C_{i j k l}^{H}=\frac{1}{|Y|} \int_{Y} C_{p q r s}\left(\varepsilon_{p q}^{0(k l)}-\varepsilon_{p q}^{*(k l)}\right)\left(\varepsilon_{r s}^{0(i j)}-\varepsilon_{r s}^{*(i j)}\right) d Y
$$


The unit cell is discretized into $n_{e}$ equal-sized elements, the independent constitutive parameters can be written as:

$$
C_{i j k l}^{H}=\sum_{i=1}^{n_{e}} Q_{i j k l}^{e}
$$

where $Q_{i j k l}^{e}$ are the element mutual energies and can be defined as

$$
Q_{i j k l}^{e}=\frac{1}{\left|Y^{e}\right|} \int_{Y^{e}} C_{p q r s}\left(\varepsilon_{p q}^{0(k l)}-\varepsilon_{p q}^{*(k l)}\right)\left(\varepsilon_{r s}^{0(i j)}-\varepsilon_{r s}^{*(i j)}\right) d Y^{e}
$$

where $Y^{e}$ is the domain of the element $e$-th in the unit cell.

\subsection{Design of linear cellular structures using inverse ho- mogenization (Step 4)}

The result of the multi-objective, metamodel-based global optimization problem (5) is the optimal volume fractions $\boldsymbol{\mu}^{*}$ of the cellular meterials within the structure. For isotropic elastic materials, the stiffness tensor $\mathbf{C}^{*}\left(\mu_{k}^{*}\right)$ can be determined using two material properties: Young's modulus $\mathrm{E}^{*}\left(\mu_{k}^{*}\right)$ and Poisson's ratio $v_{0}$. As customary in topology optimization, the Poisson's ratio is treated as constant, while the Young's modulus is defined as function of the volume fraction $\mu_{k}^{*}$. This work uses the rule of mixture model defined by

$$
\mathrm{E}^{*}\left(\mu_{k}^{*}\right)=\mathrm{E}_{\min }^{*}+\left(\mathrm{E}_{0}^{*}-\mathrm{E}_{\min }^{*}\right)\left(\mu_{k}^{*}\right)^{\eta},
$$

where $\eta \geq 1$ is a penalization power, which by default takes the value $\eta=3$. The goal of this step is to find the optimal cellular material topology with a homogenized property tensor equal (or as close as possible) to $\mathbf{C}_{k}^{*}\left(\mu_{k}^{*}\right)$. To this end, inverse homogenization is implemented $[27,28]$ to synthesize the topology of the corresponding unit cell within the cellular material. The inverse homogenization problem is stated as follows:

$$
\begin{aligned}
\text { given } & \mu_{k}^{*}, \mathbf{C}_{k}^{*}\left(\mu_{k}^{*}\right) \\
\text { find } & \mathbf{x}_{e} \in \mathbb{R}^{n_{e}} \\
\text { minimize } & f_{e}\left(\mathbf{x}_{e}\right)=\left\|\mathbf{C}_{k}^{H}\left(\mathbf{x}_{e}\right)-\mathbf{C}_{k}^{*}\left(\mu_{k}^{*}\right)\right\|^{2} \\
\text { subject to } & h_{e}\left(\mathbf{x}_{e}\right)=\frac{1}{n_{e}} \sum_{j=1}^{n_{e}} x_{j e}-\mu_{k}^{*}=0 \\
& \mathbf{0} \leqslant \mathbf{x}_{e} \leqslant \mathbf{1},
\end{aligned}
$$

where $\mathbf{C}_{k}^{H}\left(\mathbf{x}_{e}\right)$ is the homogenized material property tensor of the $k$-th cellular material, which can be calculated using (17), and $\mathbf{x}_{e}=\left[x_{1 e}, \ldots, x_{n_{e} e}\right]^{\mathrm{T}}$ is the vector of element densities within the $e$-th unit cell of the $k$-th cellular material. The optimization problem (21) is solved for each of the $K$ cellular materials so that $K$ unit cell topologies are obtained.

\section{Numerical examples}

In this section, three designs for crashworthiness are presented. The first two examples consider elastic material model, therefore, the optimal cellular material unit cell can be synthesized using inverse homogenization. For the third example, nonlinear material models are considered. In this example, the cellular material has a predefined, parameterized topology. The cellular material design step is incorporated into the metamodel-based global optimization through the mean-field homogenization method.

\subsection{D linear bumper-like structure}

This example considers a rectangular plate fixed at both ends that is impacted in the middle by a rigid object. The corresponding design problem is illustrated in Fig. 1. 2D linear finite element analysis under a static load is applied. Due to the symmetry of the problem, only half of the entire structure is analyzed. The design domain is discretized into $100 \times 30$ Q4 finite elements. Unit forces are applied to the upper-right corner of the plate. As in other academic problems in topology optimization [27], the base material has dimensionless Young's modulus $\mathrm{E}_{0}=1$ and Poisson's ratio $v_{0}=0.3$.

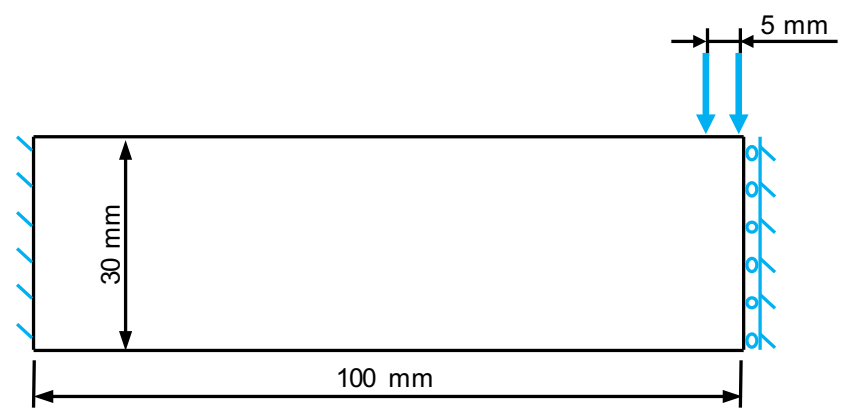

Fig. 1: Design domain for the 2D bumper problem

Step 1: Conceptual design The conceptual design is generated from the solution of the following minimum compliance, topology optimization problem:

$$
\begin{aligned}
\text { find } & \mathbf{x} \in \mathbb{R}^{n} \\
\text { minimize } & f(\mathbf{x}, \mathbf{U}(\mathbf{x}))=\mathbf{F}^{\mathrm{T}} \mathbf{U}(\mathbf{x}) \\
\text { subject to } & h(\mathbf{x})=\frac{1}{n} \sum_{e=1}^{n} x_{e}-m_{f}=0 \\
& 0 \leqslant x_{e} \leqslant 1, \quad e=1, \ldots, n,
\end{aligned}
$$

satisfying the equilibrium condition $\mathbf{K}(\mathbf{x}) \mathbf{U}(\mathbf{x})=\mathbf{F}$, where $\mathbf{K}(\mathbf{x})$ is the global stiffness matrix, $\mathbf{U}(\mathbf{x})$ is the global displacement vector, $\mathbf{F}$ is the global force vector, and $m_{f}$ is the mass fraction target. The Voigt upper bound material mixture model (2) is utilized to determine the Young's modulus in each finite element. The solution is obtained using the 
MATLAB program top88 [41]. The final topology is obtained after 48 iterations and contains 2563 unique values in $\mathbf{x}^{*}$ (Fig. 2).

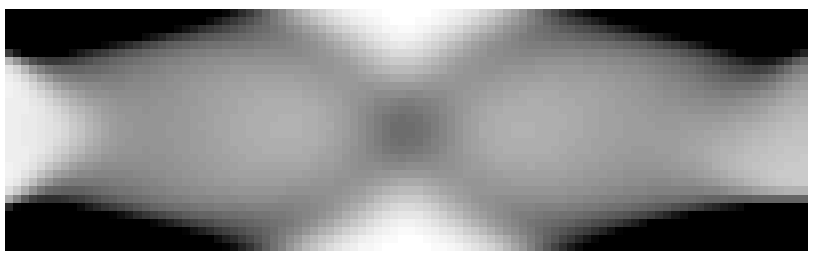

Fig. 2: Conceptual design for the 2D bumper problem, $f=$ $1.77 \times 10^{3}$.

Step 2: Clustering The conceptual design is generated using 3000 design variables with 2563 unique values. It is, therefore, impractical to utilize traditional optimization schemes due to the large number of design variables. The large number of design variables also compromises the manufacturability of the design. To overcome these problems, $\mathrm{K}$-means is used to reduce the dimension of the design space (Sec. 2.2).

A parametric study is performed to determine the influence of the $K$ value. To enhance manufacturability as well as effective use of metamodels in the next step, the optimal value is selected in the range of 1 and 10. Figure 3 shows the clustered designs corresponding to $K=1, \ldots, 10$. As the number of clusters increases, the objective value $J$ tends to decrease (see Fig. 4). For the clustered design, it is desirable to keep the lowest $K$ value as well as the lowest $J$ value. From these results, an elbow is observed at $K=4$. After this point, the rate of improvement of the objective function $J$ is significantly reduced. Therefore, $K=4$ is identified as the optimal number of clusters.

Step 3: Metamodel-based global optimization The design optimization problem in this step is to find the optimal volume fractions $\boldsymbol{\mu}$ of the cellular materials that minimize the structural compliance satisfying a mass constraint. In order to map the cellular material volume fraction to its material property, this step uses the material mixture rule (20). To ensure the feasibility of the cellular material property, $\eta=3$ is used so that the (elastic) properties fall within the HashinShrikman bounds. The problem statement is the same as the one in the conceptual design step (22), except that objective function is replaced by its Kriging metamodel. This is,

$$
\begin{aligned}
\text { find } & \boldsymbol{\mu} \in \mathbb{R}^{K}(K=4) \\
\text { minimize } & \hat{f}(\mathbf{x}(\boldsymbol{\mu})): \text { Kriging compliance } \\
\text { subject to } & h(\mathbf{x}(\boldsymbol{\mu}))=\frac{1}{n} \sum_{k=1}^{K} \sum_{x_{e} \in S_{k}} \mu_{k}-m_{f}=0 \\
& 0.1 \leqslant \mu_{k} \leqslant 1.0 \\
& k=1, \ldots, K .
\end{aligned}
$$

Note that in (23), the mass constraint $h(\mathbf{x}(\boldsymbol{\mu}))$ is a linear function, therefore, a metamodel is not required. The number of samples for LHS to build the initial metamodel $\hat{f}$ is ten times the number of clusters, i.e., $P=10 \mathrm{~K}$. The metamodel is sequentially updated using the EGO framework. The final optimal design is found in 88 iterations with the objective value $f=\hat{f}=1.80 \times 10^{3}$ and $\boldsymbol{\mu}=[0.10,0.41,0.51,1.00]^{\mathrm{T}}$. The final design uses $87 \%$ fewer design variables than the conceptual design; however, its compliance is only $1.6 \%$ higher. The next step aims to find the optimal cellular structure within each of the four cellular material clusters.

Step 4: Cellular material design For any given cellular material volume fraction, one can find its corresponding material property tensor, which is defined as the target material property tensor $\mathbf{C}^{*}\left(\mu_{k}^{*}\right)$. The goal of this step is to find the optimal cellular unit cell topology that matches the target material property $\mathbf{C}^{*}\left(\mu_{k}^{*}\right)$. The optimal cellular material volume fractions found in the previous step are $\boldsymbol{\mu}=[0.10,0.41,0.51,1.00]^{\mathrm{T}}$. The equivalent cellular structure for $\mu=1.00$ and $\mu=0.10$ are solid and void. Therefore, only the optimal cellular structures with $\mu=0.41$ and $\mu=0.51$ need to be solved using the inverse homogenization problem stated in (21). Table 1 shows the resulting optimal cellular materials for the four clusters. Figure 5 shows the optimal cellular structure of the (half domain) 2D bumperlike design by substituting the cellular unit cell from Table 1 into the clustered design (Fig. 3, $K=4$ ).

\subsection{D geometrically nonlinear bumper-like structure}

A symmetric 3D bumper-like structure is considered in this problem (Fig. 6). The structure is subjected to rigid pole impact. The mass of the impacting pole is $2.2 \times 10^{-3}$ ton and the impacting speed is $10 \times 10^{3} \mathrm{~mm} / \mathrm{s}$. The design domain is $800 \mathrm{~mm} \times 100 \mathrm{~mm} \times 80 \mathrm{~mm}$ and it is discretized into $5 \mathrm{~mm}$ $\times 5 \mathrm{~mm} \times 5 \mathrm{~mm}$ brick elements. The material of the structure is aluminum. The material properties are summarized in Table 2. The four steps involved in the optimal design of the cellular structure are described below.

Step 1: Conceptual design The conceptual design corresponds to the solution of a (linear) minimum compliance problem subjected to a mass fraction constraint of $25 \%$ [42]. In this initial problem, the impact load is replaced by static nodal forces along the center of the design domain. The solution is obtained using top $3 \mathrm{~d}$ and the material mixture model 
$\mathrm{K}=1$

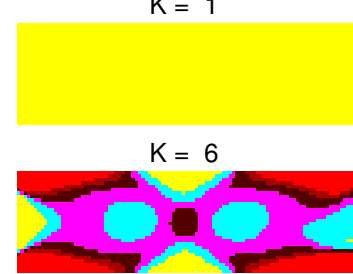

$K=2$

$\mathrm{K}=7$

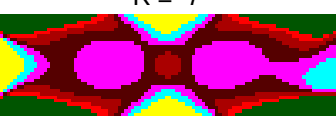

$K=3$

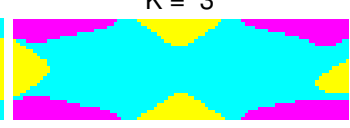

$\mathrm{K}=8$

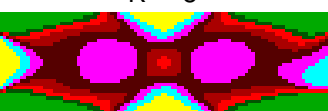

$K=4$

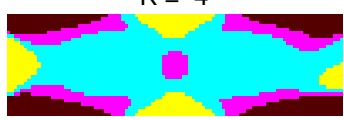

$\mathrm{K}=9$

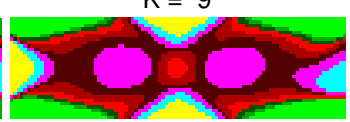

$\mathrm{K}=5$

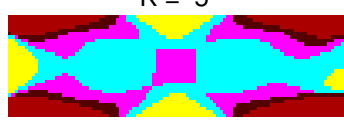

$\mathrm{K}=10$

Fig. 3: Clustered designs with $K=1 \ldots 10$.

Table 1: Optimal cellular materials for the 2D bumper problem.

\begin{tabular}{|c|c|c|c|c|c|}
\hline$k$ & $\mu_{k}^{*}$ & $\mathbf{C}_{k}^{*}\left(\mu^{*}\right)$ & $\mathbf{C}_{k}^{H}\left(\mathbf{x}_{e}^{*}\right)$ & Unit cell & Tile $3 \times 3$ \\
\hline \multirow{3}{*}{$\square 1$} & \multirow{3}{*}{0.10} & 0.0010 .0000 .000 & 0.0010 .0000 .000 & & \\
\hline & & 0.0000 .0010 .000 & 0.0000 .0010 .000 & & \\
\hline & & $\begin{array}{llll}0.000 & 0.000 & 0.000\end{array}$ & $\begin{array}{lll}0.000 & 0.000 & 0.000\end{array}$ & & \\
\hline \multirow{3}{*}{$\square 2$} & \multirow{3}{*}{0.41} & {$\left[\begin{array}{lll}0.076 & 0.023 & 0.000\end{array}\right.$} & {$\left[\begin{array}{lll}0.076 & 0.023 & 0.000\end{array}\right]$} & & \\
\hline & & 0.0230 .0760 .000 & 0.0230 .0760 .000 & & \\
\hline & & 0.0000 .0000 .027 & 0.0000 .0000 .026 & & \\
\hline \multirow{3}{*}{$\square 3$} & \multirow{3}{*}{0.51} & {$\left[\begin{array}{lll}0.149 & 0.045 & 0.000\end{array}\right]$} & {$\left[\begin{array}{lll}0.149 & 0.045 & 0.000\end{array}\right]$} & & \\
\hline & & 0.0450 .1490 .000 & 0.0450 .1490 .000 & & \\
\hline & & {$\left[\begin{array}{lll}0.000 & 0.000 & 0.052\end{array}\right]$} & $\left.\begin{array}{llll}0.000 & 0.000 & 0.052\end{array}\right]$ & & \\
\hline \multirow{3}{*}{ - 4} & \multirow{3}{*}{1.00} & {$\left[\begin{array}{lll}1.099 & 0.330 & 0.000\end{array}\right.$} & {$\left[\begin{array}{lll}1.099 & 0.330 & 0.000\end{array}\right.$} & & \\
\hline & & 0.3301 .0990 .000 & 0.3301 .0990 .000 & & \\
\hline & & $\begin{array}{llll}0.000 & 0.000 & 0.385\end{array}$ & $\left.\begin{array}{llll}0.000 & 0.000 & 0.385\end{array}\right]$ & & \\
\hline
\end{tabular}

Table 2: Base material properties.

\begin{aligned} & \hline Property Value \\ & \hline Density $2.70 \times 10^{-9}$ ton $/ \mathrm{mm}^{3} \\ &$ Elastic modulus $70 \times 10^{3} \mathrm{MPa} \\ &$ Poisson's ratio 0.33 \\ & \hline\end{aligned}

(2) [42]. The boundary conditions and the resulting conceptual design are shown in Fig. 7. This design contains 25600 elements with 23685 distinct design variable values.

Step 2: Clustering The conceptual design is clustered using $\mathrm{K}$-means with $K=1, \ldots, 10$. Figure 8 shows the resulting clustered designs. Figure 9 shows the relation between the number of clusters and the K-means objective function defined in (3). The elbow is observed at $K=3$ and this val- ues is chosen as the optimal number of clusters.

Step 3: Metamodel-based global optimization In this step, the optimization problem is to find the Pareto designs that maximize internal energy and minimize the mass of the structure. As before, the design variable $\mu_{k}$ is mapped to the material property tensor $\mathbf{C}_{k}$ using the material mixture model (20) with $\eta=3$. The multiobjective optimization problem is formulated as follows:

$$
\begin{aligned}
\text { find } & \boldsymbol{\mu} \in \mathbb{R}^{K}(K=3) \\
\text { maximize } & \hat{f}_{1}(\mathbf{x}(\boldsymbol{\mu})): \text { Kriging internal energy } \\
\text { minimize } & f_{2}(\mathbf{x}(\boldsymbol{\mu})): \text { mass fraction } \\
\text { subject to } & 0.3 \leqslant \mu_{k} \leqslant 1.0 \\
& k=1, \ldots, K .
\end{aligned}
$$

Notably, (24) has higher lower bound than (23). This 


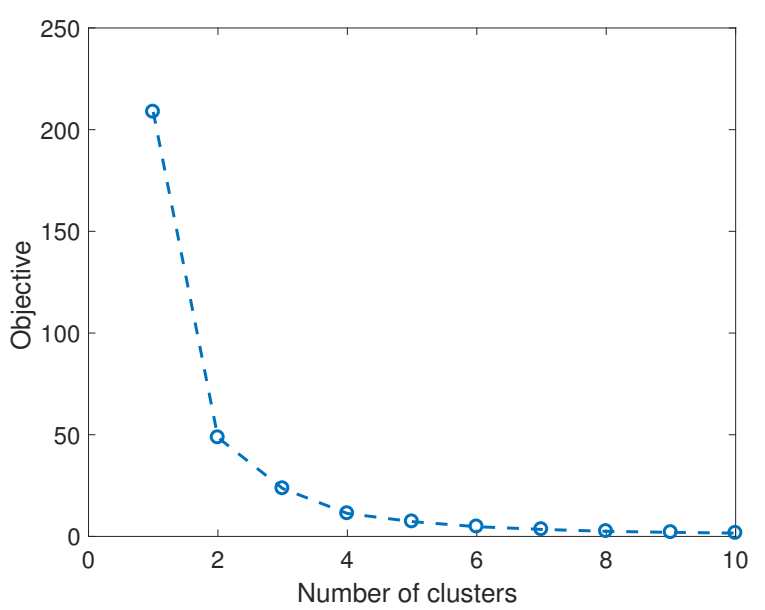

Fig. 4: K-means objective as a function of the number of clusters $K$ for the 2D bumper-like problem.

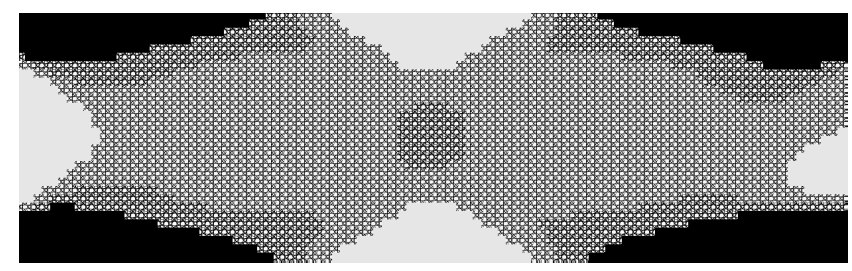

Fig. 5: Optimal cellular structure with $K=4$.

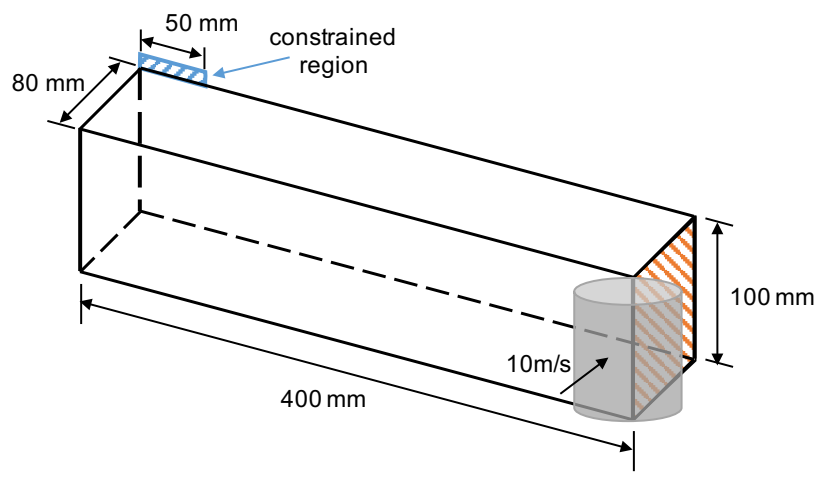

Fig. 6: Design domain for the bumper design problem

is due to the effect in the finite element model. In our experience, nonlinear models require a higher lower bound than linear models, i.e., a cellular material with a very small volume fraction $\mu_{k}$ causes numerical instabilities during the crash simulation. The Pareto designs are obtained using linear and geometrically nonlinear finite element analysis. Figure 10 shows the specific energy absorption (internal energy divided by mass fraction) contour plot, conceptual design, and the resulting Pareto fronts. As expected, the Pareto designs dominate the conceptual design (Fig. 7). Interestingly, the Pareto designs using linear finite element analysis seem to dominate the geometrically nonlinear finite element analysis counterparts. This is due to the over estimation of the in-

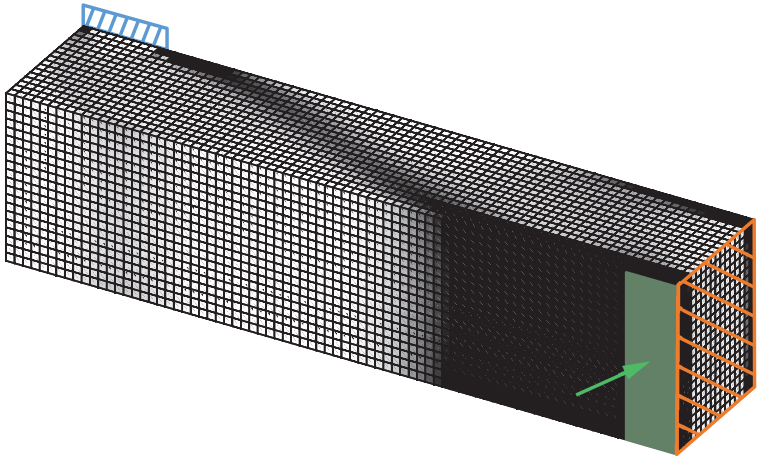

Fig. 7: The final topology generated for the bumper problem with internal energy $=1.90 \times 10^{6} \mathrm{~J}$ and mass fraction $=0.50$.

ternal energy caused by the linear approximation of the strain field.

Step 4: Cellular material design In this step, the optimal topology of the cellular unit cell of each cluster is synthesized by solving the inverse homogenization problem stated in (21). The initial design is a solid cube with a void voxel in the middle. Table 3 shows the final cellular topologies of five selected Pareto designs from both linear and geometrically nonlinear elastic models. Several topologies show a solid cube with an internal, spherical void. Random initial design would result in different topologies. As observed, even for similar structural mass values $f_{2}$, the topologies of the linear and the geometrically nonlinear cellular unit cells are different as well as the optimal cellular material volume fractions $\boldsymbol{\mu}^{*}$. Material nonlinearity is considered in Sec. 4.3.

\subsection{D nonlinear bumper-like structure}

In order to synthesize the topology of the cellular unit cells in nonlinear material models, the inverse homogenization method (Sec. 3.3) is replaced by the mean-field homogenization method (Sec. 3.1). To this end, the cellular unit cell is parameterized. In this is study, the parameter is defined as the radius of an internal, spherical void in the center of a solid cube. The cellular structure volume fraction changes as a function of this parameter, hence, the cellular material properties also changes. Figure 11 shows a set of material nonlinear response curves as a function of the volume fraction of the cellular unit cell.

The generation of the conceptual design (Step 1) and clustering (Step 2) are the same as presented in Sec. 4.2. Since the cellular unit cell topologies are already pre-determined, only metamodel-based global optimization (Step 3) is required. In this step, the material mixture rule (20) is replaced by the mean-field homogenization method on a predefined parameterized cellular structure. The metamodel-based global optimization problem is stated as 

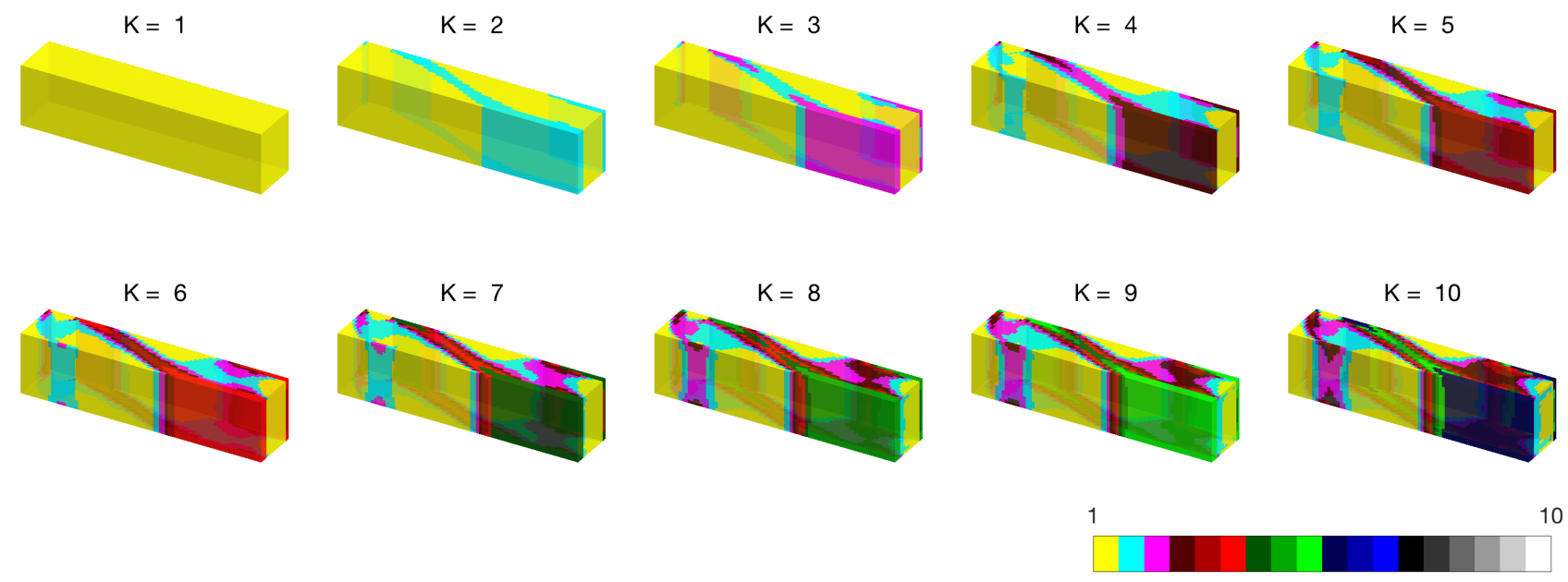

Fig. 8: Clustered design with $K=1 \ldots 10$

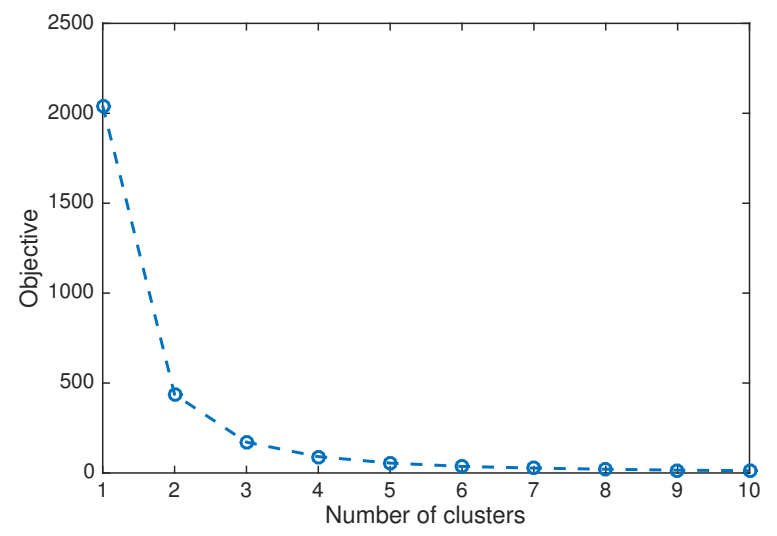

Fig. 9: K-means objective as a function of the number of clusters $K$ for the 3D bumper-like problem.

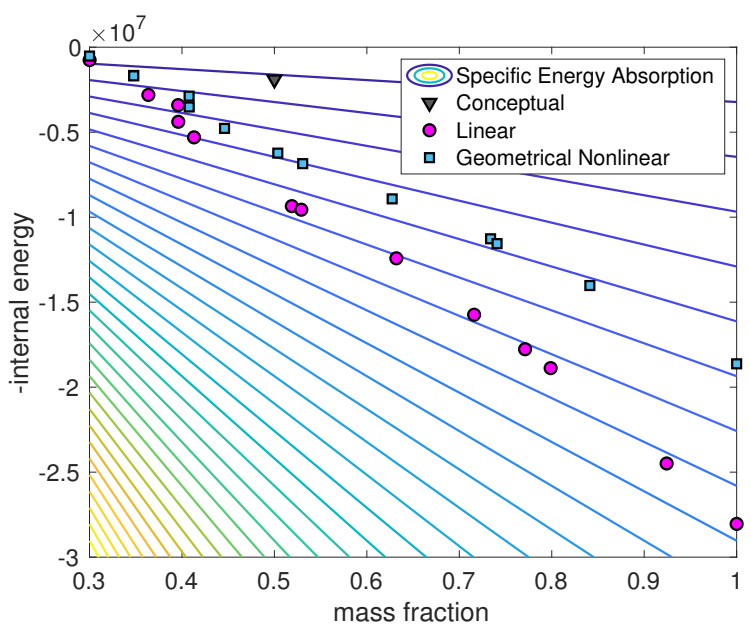

Fig. 10: Design optimization results with $K=3$.

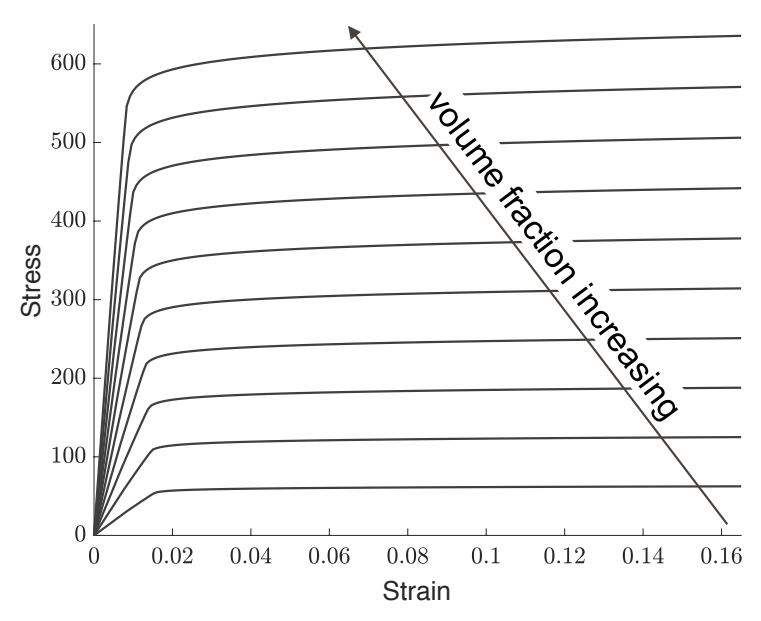

Fig. 11: Material nonlinear responses with volume fraction from 0.1 to 1.0 with step 0.1 .

$$
\begin{aligned}
\text { given } & \boldsymbol{\mu}(r), \mathbf{C}^{H}(\boldsymbol{\mu}(r)) \\
\text { find } & \mathbf{r} \in \mathbb{R}^{K}(K=3) \\
\text { maximize } & \hat{f}_{1}(\mathbf{x}(\boldsymbol{\mu}(\mathbf{r}))): \text { Kriging internal energy } \\
\text { minimize } & f_{2}(\mathbf{x}(\boldsymbol{\mu}(\mathbf{r}))): \text { mass fraction } \\
\text { subject to } & 0.3 \leqslant \mu\left(r_{k}\right) \leqslant 1.0 \\
& k=1, \ldots, K
\end{aligned}
$$

where $\mathbf{r}$ is a vector of the void sphere radius in the cellular material and $\mathbf{C}^{H}(\mu(r))$ is the homogenized cellular material property obtained by the mean-field homogenization method. Note that, the cellular material volume fraction $\mu$ now is a function of the sphere radius $r$.

The optimization problem (25) requires more computation power to solve since mean-field homogenization is re- 
Linear
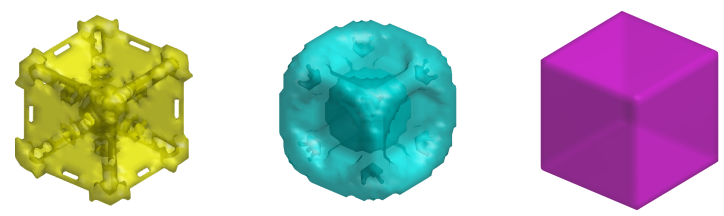

$f_{2}=0.41$ with $\boldsymbol{\mu}^{*}=[0.30,0.57,1.00]^{\mathrm{T}}$
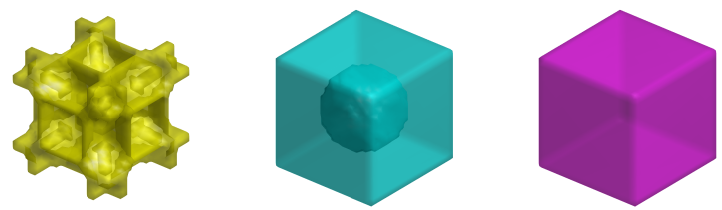

$$
f_{2}=0.53 \text { with } \boldsymbol{\mu}^{*}=[0.40,0.84,1.00]^{\mathrm{T}}
$$
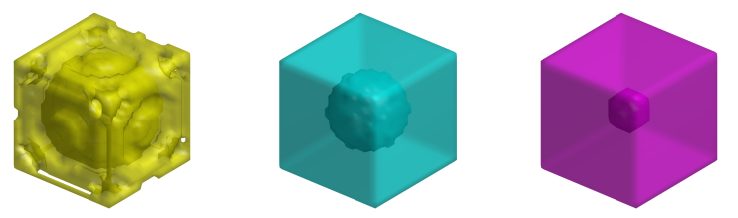

$$
f_{2}=0.63 \text { with } \boldsymbol{\mu}^{*}=[0.54,0.85,0.98]^{\mathrm{T}}
$$
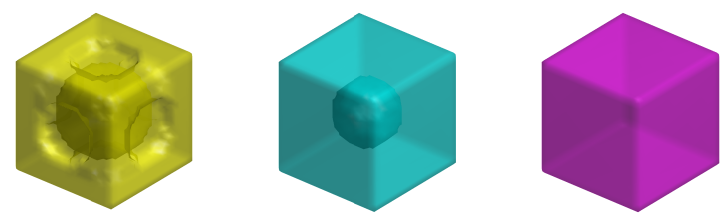

$$
f_{2}=0.72 \text { with } \boldsymbol{\mu}^{*}=[0.64,0.92,1.00]^{\mathrm{T}}
$$
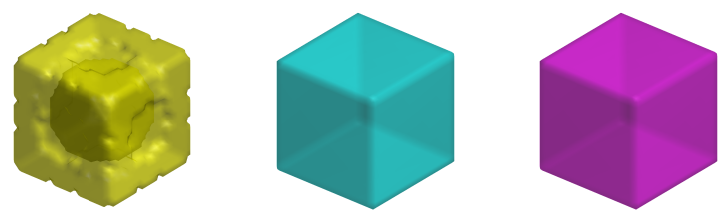

$$
f_{2}=0.80 \text { with } \boldsymbol{\mu}^{*}=[0.73,1.00,1.00]^{\mathrm{T}}
$$

Geometrically Nonlinear
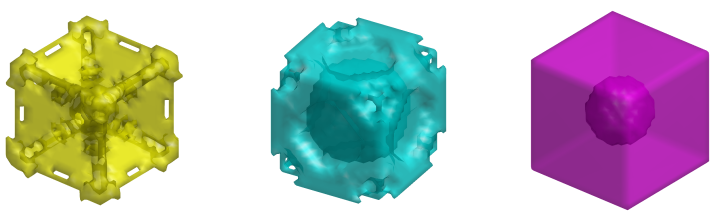

$$
f_{2}=0.41 \text { with } \boldsymbol{\mu}^{*}=[0.30,0.59,0.92]^{\mathrm{T}}
$$
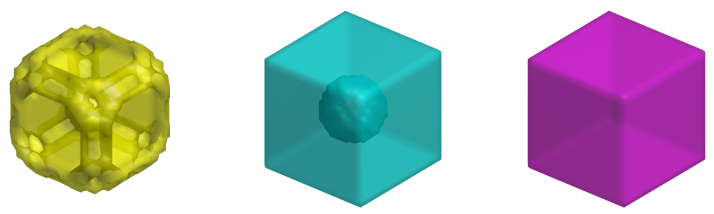

$$
f_{2}=0.53 \text { with } \boldsymbol{\mu}^{*}=[0.39,0.92,1.00]^{\mathrm{T}}
$$
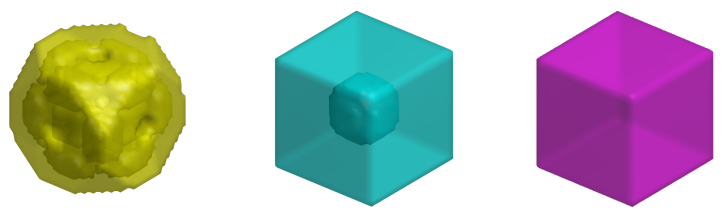

$$
f_{2}=0.63 \text { with } \boldsymbol{\mu}^{*}=[0.52,0.92,1.00]^{\mathrm{T}}
$$
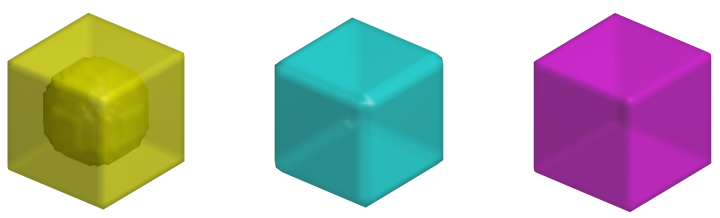

$$
f_{2}=0.74 \text { with } \boldsymbol{\mu}^{*}=[0.66,0.97,1.00]^{\mathrm{T}}
$$
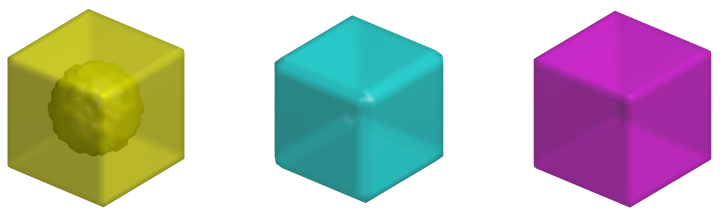

$$
f_{2}=0.84 \text { with } \boldsymbol{\mu}^{*}=[0.79,0.97,1.00]^{\mathrm{T}}
$$

quired for each EGO iteration. However, no additional cellular material design step is required since the cellular unit cell is parameterized by $r$. The comparisons of the conceptual design (Fig. 7), the resulting cellular structures obtained by the linear elastic analysis (Sec. 4.2), geometrically nonlinear elastic analysis (Sec. 4.2), nonlinear inelastic analysis, and the specific energy absorption (internal energy divided by mass fraction) contour are shown in Fig. 12. As expected, the Pareto designs dominate the conceptual design. Notably, the nonlinear analysis dominates all other designs, even with the sub-optimal cellular unit cell. This indicates the importance of considering a nonlinear model in the design. 


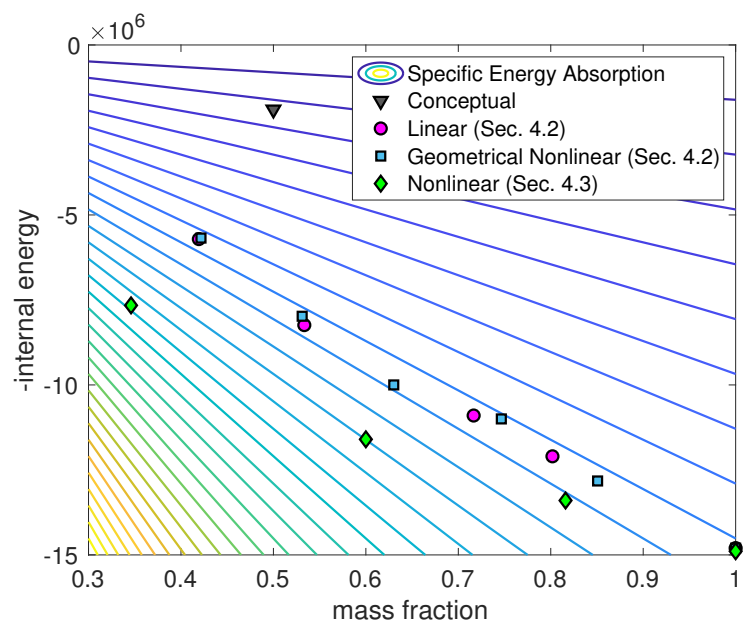

Fig. 12: Comparison of the Pareto optimal cellular structures.

\section{Summary and Discussion}

This work introduces a cluster-based optimization method for the design cellular materials and structures. The proposed method consists of four steps: conceptual design generation, clustering, metamodel-based global optimization and cellular material design. The conceptual design consists of a continuous distribution of a response variable or a design variable generated through the solution of a structural optimization problem. The K-means clustering algorithm is utilized to reduce the dimension of the optimization problem from thousands of design variables to a tractable number of cellular material clusters. With the reduced number of design variables, metamodel-based global optimization algorithms, such as EGO, can be utilized. For the design of linear and geometrically nonlinear material models, inverse homogenization is implemented so that the optimal cellular topologies for each cluster are obtained.

For (fully) nonlinear material models, the cellular material design step is incorporated into the metamodel-based global optimization through the mean-field homogenization method. The benefits of the proposed method, particularly handling nonlinear models, are demonstrated through design problems for crashworthiness that incorporate three different material models, namely, 2D linear, 3D geometry nonlinear, and 3D (fully) nonlinear. The resulting cellular structures are evaluated under a dynamic crushing load. Simplifications and assumptions by using either linear static finite element analysis or elastic material will over-predict the structure performance during the optimization. As the structures are not optimized under the nonlinear dynamic cursing load, they are dominated by the designs that are optimized under the nonlinear dynamic crushing load.

While the proposed method can effectively handle the large-scale, nonlinear cellular structures undergoing crushing load, it has limitations that are currently under ongoing research. With respect to the material model: densification in the cellular materials and material failure need to be incorporated. With respect to the design method, the applica- tion of the mean-field homogenization method is limited to predefined parameterized cellular material models; inverse homogenization-like methods are currently under investigation.

\section{Acknowledgements}

Honda R\&D Americas supported this research effort. Any opinions, findings, conclusions, and recommendations expressed in this investigation are those of the writers and do not necessarily reflect the views of the sponsors.

\section{References}

[1] Gibson, L. J., and Ashby, M. F., 1997. Cellular Solids: Structure and Properties, 2 ed. Cambridge Solid State Science Series. Cambridge University Press.

[2] Gibson, L. J., Ashby, M. F., and Harley, B. A., 2010. Cellular materials in nature and medicine. Cambridge University Press.

[3] Wu, T., Liu, K., and Tovar, A., 2017. "Multiphase topology optimization of lattice injection molds". Computers \& Structures, 192, pp. 71-82.

[4] Mehta, P. S., Solis Ocampo, J., Tovar, A., and Chaudhari, P., 2016. "Bio-inspired design of lightweight and protective structures". In SAE 2016 World Congress and Exhibition, SAE International.

[5] Orringer, O., Knadle, K. T., and Mandell, J. F., 1984. "Crash padding mechanical properties and impact response analysis". In SAE Government Industry Meeting and Exposition, SAE International.

[6] Cui, L., Kiernan, S., and Gilchrist, M. D., 2009. "Designing the energy absorption capacity of functionally graded foam materials". Materials Science and Engineering: A, 507(1-2), may, pp. 215-225.

[7] Yin, H., Wen, G., Liu, Z., and Qing, Q., 2014. "Crashworthiness optimization design for foam-filled multicell thin-walled structures". Thin-Walled Structures, 75(0), pp. $8-17$.

[8] Bi, J., Fang, H., Wang, Q., and Ren, X., 2010. "Modeling and optimization of foam-filled thin-walled columns for crashworthiness designs". Finite Elements in Analysis and Design, 46(9), pp. 698-709.

[9] Zhang, Z., Liu, S., and Tang, Z., 2011. "Comparisons of honeycomb sandwich and foam-filled cylindrical columns under axial crushing loads". Thin-Walled Structures, 49(9), pp. 1071-1079.

[10] Paz, J., Díaz, J., Romera, L., and Costas, M., 2015. "Size and shape optimization of aluminum tubes with gfrp honeycomb reinforcements for crashworthy aircraft structures". Composite Structures, 133, pp. 499507.

[11] Ahmad, Z., and Thambiratnam, D., 2009. "Crushing response of foam-filled conical tubes under quasi-static axial loading”. Materials \& design, 30(7), pp. 23932403.

[12] Yin, H., Fang, H., Xiao, Y., Wen, G., and Qing, Q., 2015. "Multi-objective robust optimization of foam- 
filled tapered multi-cell thin-walled structures". Structural and Multidisciplinary Optimization, jul.

[13] Ahmad, Z., and Thambiratnam, D. P., 2009. "Dynamic computer simulation and energy absorption of foamfilled conical tubes under axial impact loading". Computers \& Structures, 87(3-4), pp. 186-197.

[14] Correa, D. M., Klatt, T., Cortes, S., Haberman, M., Kovar, D., and Seepersad, C., 2015. "Negative stiffness honeycombs for recoverable shock isolation". Rapid Prototyping Journal, 21(2), pp. 193-200.

[15] Correa, D. M., Seepersad, C. C., and Haberman, M. R., 2015. "Mechanical design of negative stiffness honeycomb materials". Integrating Materials and Manufacturing Innovation, 4(1), Jul, p. 10.

[16] Cortes, S., Allison, J., Morris, C., Haberman, M., Seepersad, C., and Kovar, D., 2017. "Design, manufacture, and quasi-static testing of metallic negative stiffness structures within a polymer matrix". Experimental Mechanics, 57(8), pp. 1183-1191.

[17] Matthews, J., Klatt, T., Morris, C., Seepersad, C. C., Haberman, M., and Shahan, D., 2016. "Hierarchical design of negative stiffness metamaterials using a bayesian network classifier". Journal of Mechanical Design, 138(4), p. 041404.

[18] Bendsøe, M. P., and Kikuchi, N., 1988. "Generating optimal topologies in structural design using a homogenization method". Computer Methods in Applied Mechanics and Engineering, 71(2), pp. 197-224.

[19] Rodrigues, H. C., Guedes, J. M., and Bendsøe, M. P., 2002. "Hierarchical optimization of material and structure". Structural and Multidisciplinary Optimization, 24(1), pp. 1-10.

[20] Coelho, P. G., Fernandes, P. R., Guedes, J. M., and Rodrigues, H. C., 2008. "A hierarchical model for concurrent material and topology optimisation of threedimensional structures". Structural and Multidisciplinary Optimization, 35(2), pp. 107-115.

[21] Miehe, C., 2002. "Strain-driven homogenization of inelastic microstructures and composites based on an incremental variational formulation". International Journal for numerical methods in engineering, 55(11), pp. 1285-1322.

[22] Feyel, F., and Chaboche, J.-L., 2000. "Fe2 multiscale approach for modelling the elastoviscoplastic behaviour of long fibre sic/ti composite materials". Computer methods in applied mechanics and engineering, 183(3-4), pp. 309-330.

[23] Geers, M. G., Kouznetsova, V. G., and Brekelmans, W., 2010. "Multi-scale computational homogenization: Trends and challenges". Journal of computational and applied mathematics, 234(7), pp. 2175-2182.

[24] Xia, L., and Breitkopf, P., 2017. "Recent advances on topology optimization of multiscale nonlinear structures". Archives of Computational Methods in Engineering, 24(2), pp. 227-249.

[25] Fang, J., Sun, G., Qiu, N., Kim, N. H., and Li, Q., 2017. "On design optimization for structural crashworthiness and its state of the art". Structural and Multi- disciplinary Optimization, 55(3), Mar, pp. 1091-1119.

[26] Liu, K., Detwiler, D., and Tovar, A., 2017. "Optimal design of nonlinear multimaterial structures for crashworthiness using cluster analysis". Journal of Mechanical Design, 139(10), p. 101401.

[27] Bendsøe, M. P., and Sigmund, O., 2003. Topology optimization: theory, method and applications. Springer.

[28] Xia, L., and Breitkopf, P., 2015. "Multiscale structural topology optimization with an approximate constitutive model for local material microstructure". Computer Methods in Applied Mechanics and Engineering.

[29] Perdahcioğlu, E. S., and Geijselaers, H. J., 2011. "Constitutive modeling of two phase materials using the mean field method for homogenization". International journal of material forming, 4(2), pp. 93-102.

[30] Digimat, 2016. User Manual, version 2017.0 ed. eXstream Engineering, December.

[31] MacQueen, J. B., 1967. "Some methods for classification and analysis of multivariate observations". In 5-th Berkeley Symposium on Mathematical Statistics and Probability, Vol. 1, University of California Press, pp. 281-297.

[32] MacKay, D., 2003. Information Theory, Inference, and Learning Algorithms. Cambridge University Press, Cambridge, UK.

[33] Liu, K., Tovar, A., Nutwell, E., and Detwiler, D., 2015. "Thin-walled compliant mechanism component design assisted by machine learning and multiple surrogates". In SAE World Congress.

[34] Forrester, A. I. J., Sóbester, A., and Keane, A. J., 2008. Engineering Design via Surrogate Models. JohnWiley \& Sons, Chichester, UK.

[35] Lophaven, S. N., Nielsen, H. B., and Sondergaard, J., 2002. "dace"-a "matlab" kriging toolbox. Tech. rep., Informatics and Mathematical Modelling, Technical University of Denmark.

[36] Jones, D. R., Schonlau, M., and Welch, W. J., 1998. "Efficient global optimization of expensive black-box functions". Journal of Global Optimization, 13, pp. 455-492.

[37] Mori, T., and Tanaka, K., 1973. "Average stress in matrix and average elastic energy of materials with misfitting inclusions". Acta Metallurgica, 21(5), pp. 571 574.

[38] Eshelby, J., 1957. "The determination of the elastic field of an ellipsoidal inclusion, and related problems". Proceedings of the Royal Society of London A: Mathematical, Physical and Engineering Sciences, 241(1226), pp. 376-396.

[39] Hassani, B., and Hinton, E., 1998. Homogenization and Structural Topology Optimization: Theory, Practice and Software. Springer.

[40] Sigmund, O., 1994. "Materials with prescribed constitutive parameters-an inverse homogenization problem". International Journal of Solids and Structures, 31(17), pp. 2313-2329.

[41] Andreassen, E., Clausen, A., Schevenels, M., Lazarov, B. S., and Sigmund, O., 2011. "Efficient topology op- 
timization in MATLAB using 88 lines of code". Structural and Multidisciplinary Optimization, 43(1), pp. 116.

[42] Liu, K., and Tovar, A., 2014. "An efficient 3D topology optimization code written in Matlab". Structural and Multidisciplinary Optimization, 50(6), pp. 1175-1196. 